\title{
ARTICLE
}

Received 17 Jul 2013 | Accepted 2 Dec 2013 | Published 28 Jan $2014 \quad$ DOl: 10.1038/ncomms4044

\section{Hydrochromic molecular switches for water-jet rewritable paper}

\author{
Lan Sheng ${ }^{1}$, Minjie Li ${ }^{1}$, Shaoyin Zhu' ${ }^{1}$ Hao Li ${ }^{2}$, Guan Xi', Yong-Gang Li ${ }^{1}$, Yi Wang ${ }^{1}$,
} Quanshun $\mathrm{Li}^{3}$, Shaojun Liang ${ }^{3}$, Ke Zhong ${ }^{1} \&$ Sean Xiao-An Zhang ${ }^{1}$

The days of rewritable paper are coming, printers of the future will use water-jet paper. Although several kinds of rewritable paper have been reported, practical usage of them is rare. Herein, a new rewritable paper for ink-free printing is proposed and demonstrated successfully by using water as the sole trigger to switch hydrochromic dyes on solid media. Water-jet prints with various colours are achieved with a commercial desktop printer based on these hydrochromic rewritable papers. The prints can be erased and rewritten dozens of times with no significant loss in colour quality. This rewritable paper is promising in that it can serve an eco-friendly information display to meet the increasing global needs for environmental protection.

\footnotetext{
${ }^{1}$ State Key Laboratory of Supramolecular Structure and Materials, College of Chemistry, Jilin University, Changchun 130012, China. ${ }^{2}$ Department of Chemistry, Northwestern University, 2145 Sheridan Road, Evanston, Illinois 60208, USA. ${ }^{3}$ Key Laboratory for Molecular Enzymology and Engineering of Ministry of Education, College of Life Science, Jilin University, Changchun 130012, China. Correspondence and requests for materials should be addressed to S.X.-A.Z. (email: seanzhang@jlu.edu.cn) or to M.L. (email: liminjie@jlu.edu.cn).
} 
A lthough we live in an age dominated by electronic displays, paper prints still play a crucial role in our daily lives. Even though the initial cost of office printing setup is relatively low, actual cost of its on-going usage can be quite substantial because of frequent replacement of expensive ink cartridges. In addition, several international surveys reported that $90 \%$ of all information in businesses is currently retained on paper, and most of the prints are only used for one-time reading before being disposed ${ }^{1}$. Current digital prints do not de-ink and recycle well, and this creates huge problems of solid waste and environmental pollution. Ink-free printing and rewritable paper $^{2-10}$ have recently attracted attention owing to their potential benefits to forest conservation ${ }^{11}$, waste paper reduction and cutting down the cost of office printing. Incorporating switchable dyes in paper as an imaging layer represents an ideal economic approach towards rewritable paper. Leuco dyes ${ }^{12,13}$ and photochromic compounds ${ }^{14,15}$ have attracted much attention as new rewritable imaging dyes for rewritable paper. In particular, spiropyrans and related molecular switches, which are well known to undergo light-stimulated reversible isomerizations ${ }^{16-19}$ between ring-closed forms (CFs) and ringopen forms (OFs) accompanied by significant colour changes, might be suitable candidates for the development of rewritable paper 20,21 . However, the prevailing colour-switching stimuli for these molecules are mainly UV/Vis ${ }^{22,23}$ and acid/base ${ }^{24,25}$, which lead to problems, such as molecular degradation, potential safety issues and environmental hazards. These problems limit greatly their practical applications.

From a green perspective ${ }^{26}$, it would be ideal if water can be used as the trigger to stimulate molecular switches for the development of rewritable paper. As water is a renewable resource and obviously poses no risk to the environment, its introduction and removal can be safely achieved, and water integrates well with fully developed ink-jet printing techniques (here for convenience, the water-triggered switchable dyes are named hydrochromic dyes, and printing with water only instead of ink is called waterjet printing). However, little progress has been made in this respect because of some major challenges. Specifically, the dye molecule should (i) exist in only one of its two states in the absence or presence of water, respectively, with high preference, (ii) be capable of integration into paper-based materials, where the hydrochromic behaviour still lives on and (iii) the resulting paper materials should be stable and robust enough to endure many write and erase cycles under ambient or mild conditions.

Herein, we systemically screen three types of 18 structurally related switchable molecules in order to investigate the structurehydrochromic property relationship in solution and evaluate their potential as water-triggered imaging materials. We then investigate the performances of candidate dyes in the solid state. Finally, we develop a prototype of rewritable paper using suitable hydrochromic dyes, which integrates well with current ink-jet printing techniques and demonstrates the reversibility and stability, indicating the promising real application of these materials.

\section{Results}

Screening for suitable hydrochromic dyes. Inspired by the literature ${ }^{27-31}$ studying solvation effects on the isomerization of some spiropyrans in aqueous solutions, we conjectured that the spiropyrans and related molecular switches could be potential hydrochromic dyes. By reviewing existing solvatochromic results of spiropyrans and related molecular switches reported so far, one critical problem preventing them being used practically is the colour instability under ambient light ${ }^{27-29}$. This might be due to unfavourable conjugated push-pull zwitterionic structure of their merocyanine isomers, which readily undergoes the ring-closing reaction under visible light. We assumed that breaking the conjugated linkage of the zwitterions could improve their colour stability. In order to prove our assumption, we have designed and synthesized three classes of 18 structurally related switchable molecules-oxazolidines (1-10), oxazines (11-13) and spiropyrans (14-18) (Fig. 1a). 1-10 were synthesized ${ }^{19,32}$ with a modified four-step procedure by using phenylhydrazines as starting materials, as shown in Fig. 2. First, 2,3,3-trimethylindolenine intermediates were obtained by the classical method of Fischer indole synthesis. These indolenines are then quaternized with 2-bromoethanol to afford indoleninium bromides, which are subsequently treated with the corresponding aromatic aldehydes in ethanol with $\mathrm{CH}_{3} \mathrm{SO}_{3} \mathrm{H}$ as a catalyst to obtain precipitated condensation products. At last, the CFs of 1-10 were obtained by basic treatment of condensation products with $5 \% \mathrm{NaHCO}_{3}$ aqueous solutions and extraction with ethyl acetate. 11-13 were synthesized ${ }^{33,34}$ according to the work reported by F. M. Raymo's group and us. 14-18 were synthesized and purified according to literature $27,35,36$. The detailed synthesis and characterization information for 1-18 was supplied in Supplementary Methods.

Quick solution tests of the hydrochromic properties of 1-18 were conducted first in 1,4-dioxane/water binary solvent systems (Fig. 1b). To our pleasant surprise, most of oxazolidines (1-5) and oxazines (11-12) have favourable hydrochromic properties with good colour intensity and stability at ambient conditions, while the well-studied conventional spiropyrans (14-18) hardly show hydrochromism under the same conditions, and their OFs can only be observed either at elevated temperature or in the dark with poor colour intensity (more than one order of magnitude lower) (see Supplementary Fig. 1 and Supplementary Discussion). Furthermore, oxazolidines or oxazines can be converted exclusively to their coloured OFs with water at room temperature (see Supplementary Fig. 2).

Conjugated substituents conjoined to molecular structures usually play important roles on their properties, such as sensitivity, solubility, molecular colour and colour intensity. To verify how various substituents actually affect these properties of our molecules, a dual-correlation screening of both wavelength and intensity of the maximum absorption peak against the water content is used (see Supplementary Fig. 1). The results show that 5 and $\mathbf{1 1}$ are very labile to ambient polarity, and $\mathbf{1 2}$ has poor solubility in aqueous solution. It needs to be mentioned that a non-conjugated functional effect on molecular colour has also been discovered herein-that is, the wavelength of the maximum absorption peak red shifts in the case of $\mathbf{1 1}$ versus $\mathbf{2}$ or $\mathbf{1 2}$ versus 1 (nitrophenoxide versus ethoxide substitute on nitrogen), which have the same positively charged colour-dominating backbones in their OFs (see Supplementary Fig. 1b and Supplementary Discussion). The screening results indicate that oxazolidines with electron-donating groups on $\mathrm{R}^{2}$ and either electron-withdrawing groups on $\mathrm{R}^{1}$ or none, such as $\mathbf{1 - 4}$, are good candidates for hydrochromic dyes because of their desired properties of good solubility, fast switchability, fine-tunable chroma, excellent colour intensity and stability.

Reversible hydrochromic behaviour of $\mathbf{1}$. We take $\mathbf{1}$ as an example herein to show clearly in detail the reversible hydrochromic behaviour between its ring-closed isomer $\mathbf{1}_{\mathrm{C}}$ and ringopen isomer $\mathbf{1}_{\mathbf{O}}$ in EtOH- $\mathrm{H}_{2} \mathrm{O}$ solutions (Fig. 3a). Without water, only one broad hump absorption band can be observed at wavelengths $<440 \mathrm{~nm}$, which indicates that 1 exists completely as $\mathbf{1}_{\mathrm{C}}$ in ethanol. Upon increasing the water content (Fig. 3b), a new absorption band centred at $588 \mathrm{~nm}$ (presumably $\mathbf{1}_{\mathbf{O}}$ ) is observed 


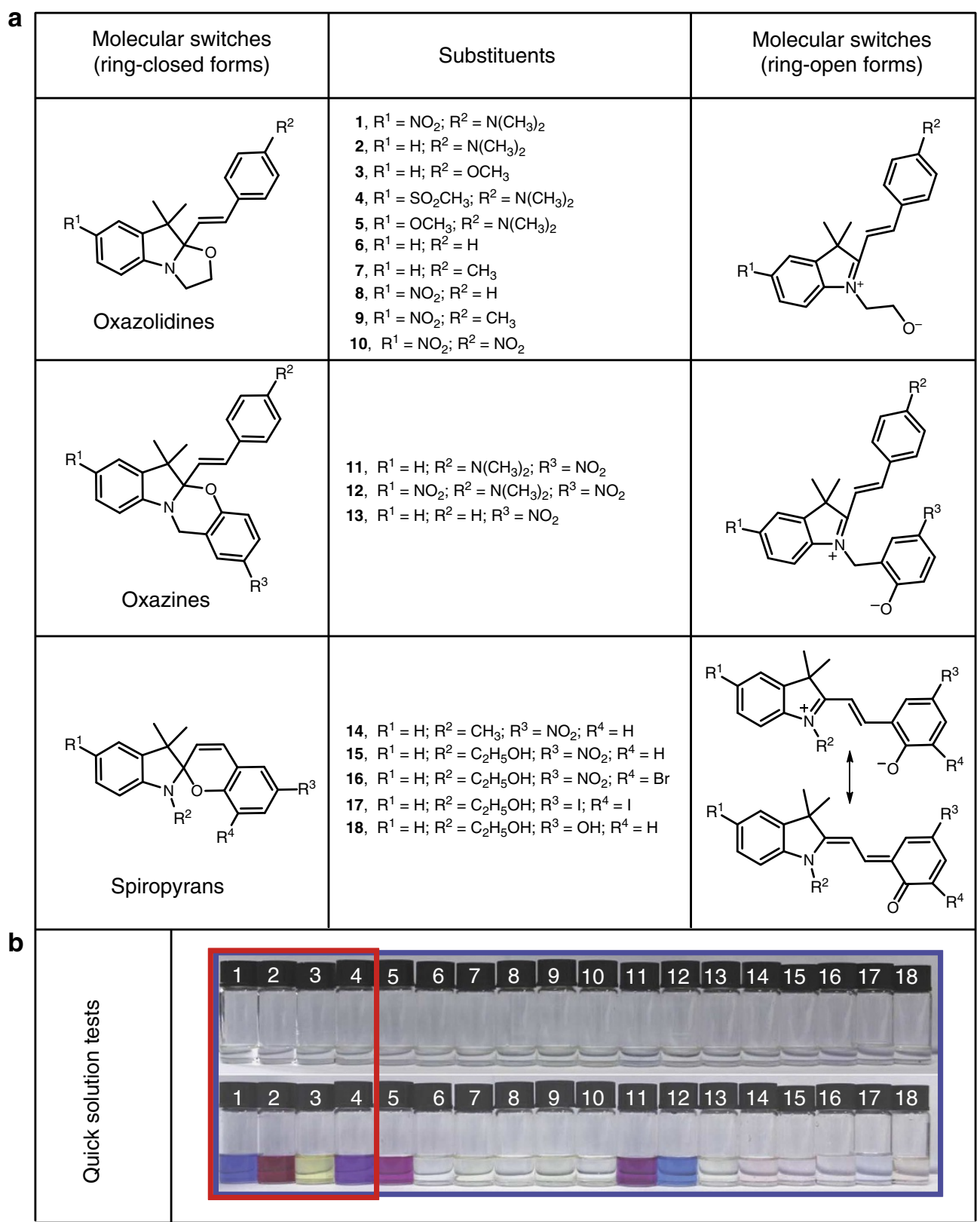

Figure 1 | Molecule design and screening for suitable hydrochromic dyes. (a) Structures of the investigated three classes of molecular switches 1-18 in their ring-closed forms and ring-open forms with different substituents and (b) the results of their quick solution tests (Photographs of solutions of 1-18 in 1,4-dioxane ( $2 \mathrm{ml}, \mathrm{C}=1 \times 10^{-4} \mathrm{moll}^{-1}$ ) before (above) and after (below) adding $2 \mathrm{ml}$ of water at ambient conditions. The suitable hydrochromic dyes are marked by red frame.

with an isosbestic point at $440 \mathrm{~nm}$ and increases along with a gradual decrease of its original hump absorption. ${ }^{1} \mathrm{H}$ NMR spectroscopy of this hydrochromic reaction also firmly supports the changes from $\mathbf{1}_{\mathbf{C}}$ to $\mathbf{1}_{\mathbf{O}}$ (see Supplementary Fig. 3 ). As the water content is decreased (see Supplementary Fig. 4), the absorption band associated with the $\mathbf{1}_{\mathrm{O}}$ at $588 \mathrm{~nm}$ gradually diminishes with the same isosbestic point. The microenvironment with multi-molecular hydrogen-bonding networks ${ }^{27,28}$ plays an important role for this water-induced switching phenomenon from $\mathbf{1}_{\mathbf{C}}$ to $\mathbf{1}_{\mathbf{O}}$, which was demonstrated by the fact that an equilibrium of both $\mathbf{1}_{\mathbf{C}}$ and $\mathbf{1}_{\mathbf{O}}$ coexsits also in some other protic solvents-that is, methanol and ethylene glycol (see Supplementary Fig. 5, Supplementary Table 1 and Supplementary Discussion). Water with both properties of a larger dipole and better hydrogen-bond donator can significantly reduce the energy of $\mathbf{1}_{\mathrm{O}}$ more than $\mathbf{1}_{\mathrm{C}}$. The energy of $\mathbf{1}_{\mathrm{O}}$ becomes lower than $\mathbf{1}_{\mathbf{C}}$ when the water content reaches a certain amount enough, to support a better solvato-microenvironment, consequently shifting equilibrium from $\mathbf{1}_{\mathbf{C}}$ to $\mathbf{1}_{\mathrm{O}}$ (see Supplementary Fig. 6). These results indicate that the water-triggered molecular switching process is in thermodynamic control. The equilibrium constant $K_{\mathrm{eq}}=0.113$ for 1 in $\mathrm{CD}_{3} \mathrm{OD} / \mathrm{H}_{2} \mathrm{O}$ has been calculated based on ${ }^{1} \mathrm{H}$ NMR data (see Supplementary Fig. 7 and Supplementary Note 1). The observation of this fully reversible hydrochromic property of $\mathbf{1}$ demonstrates its potential application as a water-triggered imaging material.

Structure design of the water-jet rewritable paper. An ethanol solution of $\mathbf{1}_{\mathbf{C}}$ was then directly soaked into a paper substrate to 

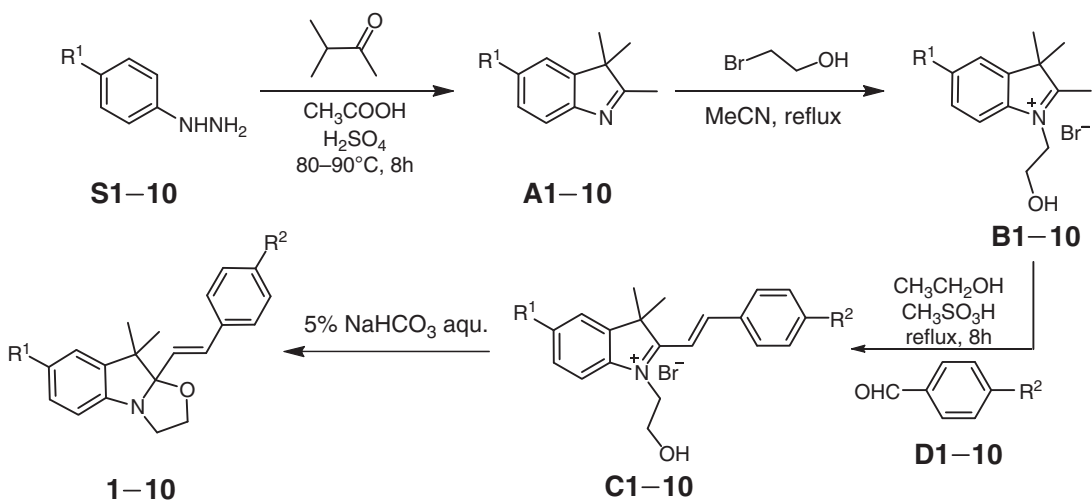
1, $R^{1}=\mathrm{NO}_{2} ; \mathrm{R}^{2}=\mathrm{N}\left(\mathrm{CH}_{3}\right)_{2}$
6, $\mathrm{R}^{1}=\mathrm{H} ; \mathrm{R}^{2}=\mathrm{H}$
2, $\mathrm{R}^{1}=\mathrm{H} ; \mathrm{R}^{2}=\mathrm{N}\left(\mathrm{CH}_{3}\right)_{2}$
7, $\mathrm{R}^{1}=\mathrm{H} ; \mathrm{R}^{2}=\mathrm{CH}_{3}$
3, $\mathrm{R}^{1}=\mathrm{H} ; \mathrm{R}^{2}=\mathrm{OCH}_{3}$
8, $\mathrm{R}^{1}=\mathrm{NO}_{2} ; \mathrm{R}^{2}=\mathrm{H}$
4, $\mathrm{R}^{1}=\mathrm{SO}_{2} \mathrm{CH}_{3} ; \mathrm{R}^{2}=\mathrm{N}\left(\mathrm{CH}_{3}\right)_{2}$
9, $\mathrm{R}^{1}=\mathrm{NO}_{2} ; \mathrm{R}^{2}=\mathrm{CH}_{3}$
5, $\mathrm{R}^{1}=\mathrm{OCH}_{3} ; \mathrm{R}^{2}=\mathrm{N}\left(\mathrm{CH}_{3}\right)_{2}$
10, $R^{1}=\mathrm{NO}_{2} ; R^{2}=\mathrm{NO}_{2}$

Figure 2 | General route for synthesis of 1-10. Preparation of oxazolidines 1-10 starts with various substituted phenylhydrazines (S1-10); they are converted to corresponding 2,3,3-trimethylindolenine (A1-10) first, indoleninium bromides (B1-10) next, condensation intermediates (C1-10) then and finally to the desired target molecules (1-10). 'aqu.' is the abbreviation for aqueous solution.

a



Closed form

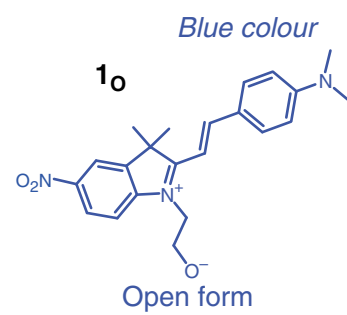

b

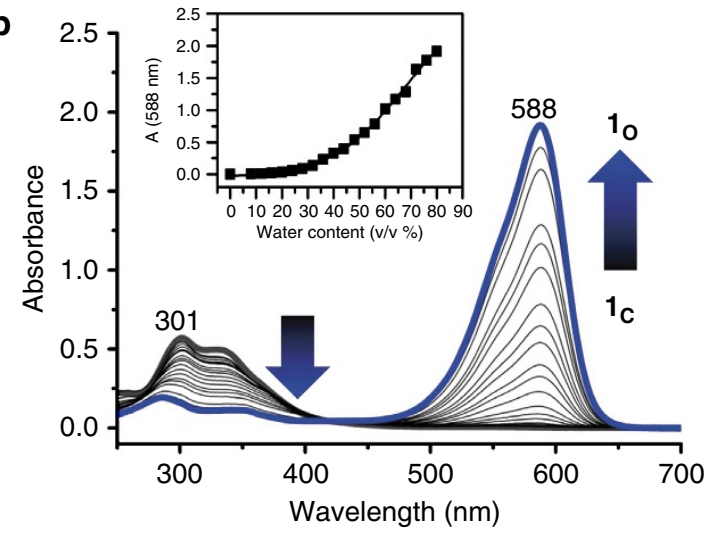

Figure 3 | Water-triggered reversible isomerization of 1 in solution.

(a) The illustration of reversible water-stimulated isomerization between ring-closed and ring-open isomers for 1. (b) UV-vis absorption spectra of $\mathbf{1}$ in variable mixtures of ethanol and water with increasing percentage of water by volume from 0 to $80 \%\left(C=2 \times 10^{-5} \mathrm{moll}^{-1}, 20^{\circ} \mathrm{C}\right.$, see Supplementary Methods). Inset: Plot of absorbance at $\lambda_{\max }=588 \mathrm{~nm}$ with water content.

further investigate whether its reversible hydrochromic behaviour still lives on in the solid state. Unfortunately, to our surprise, a stable blue colour of 1 remains on the paper after solvent evaporation even in the absence of water. The same phenomenon is observed also with other polyhydroxyl substrates, such as silica gel, and polyvinyl alcohol, but does not appear on non-hydroxyl substrates, such as glass, non-cellulose filter paper, polyester and nylon. These results indicate that microenvironments play a crucial role to stabilize thermodynamically certain isomers, and the stable blue colour of $\mathbf{1}$ is closely related with the polyhydroxyl microenvironment, which favours the $\mathbf{1}_{\mathbf{O}}$ thermodynamically. This conclusion is further supported by the experimental observation that the blue colour can be switched to colourless with base and returned back to blue with acid (see Supplementary Fig. 8). By analysing the difference between ethanol solution and the solid substrates, we hypothesize that the undesired conversion of $\mathbf{1}_{\mathrm{C}}$ to $\mathbf{1}_{\mathbf{O}}$ is caused by the presence of an immobile polyhydroxyl microenvironment and stable spatial multi-hydroxyl participation, which might provide much better stabilization to $\mathbf{1}_{\mathbf{O}}$ than the mobile multi-hydroxyl environment in ethanol. This phenomenon observed herein is conceptually similar to some cases of reactions that proceed easily in an immobile microenvironment of an enzyme but do not happen in solution. We assume that passivation of the hydrogen bonding of the polyhydroxyls of paper might be a key to solve the problem. Consistent with this hypothesis are the facts that the stable blue colour of $\mathbf{1}$ on paper can be reconverted back to colourless by post-treatment with dimethyl sulfoxide (DMSO), a hydrogen bond acceptor, and the colour of this revived $\mathbf{1}$ on the cellulose paper can be reversibly switched back with water. In addition, the unwished blue colour of $\mathbf{1}_{\mathbf{O}}$ can also be avoided when the paper substrate is pretreated with DMSO before loading $\mathbf{1}_{\mathrm{C}}$. For practical application, nonvolatile polyethylene glycol (PEG) as a hydrogen bond acceptor proved to be a better material than DMSO for modification of the paper cellulose. Other challenges preventing the dye from being used practically in the solid state were also encountered and successfully overcome, such as unwanted colouration in damp air, long colour developing time because of slow water penetration, quick fading of the colour because of fast water evaporation under relatively warm and dry conditions, and the formation of microcrystalline aggregates of $\mathbf{1}_{\mathrm{C}}$. Microcrystalline aggregates are not switchable, which is supported by the observation that the khaki powder of $\mathbf{1}_{\mathrm{C}}$ does not undergo colour change after its exposure to water.

To solve these problems, a prototype of a four-layer structure to be employed as rewritable paper, abbreviated as $\mathbf{R P}$, has been developed with the help of PEG to enhance its practical usability against various extreme real life situations. PEG not only 
passivates efficiently the hydroxyl groups of paper but also possesses excellent swellability for water, which facilitates the penetration of water molecules, and consequently increases the opportunity of triggering the ring-opening isomerization of $\mathbf{1}_{\mathrm{C}}$. In addition, PEG also acts as a solid state solvent and prevents $\mathbf{1}_{\mathbf{C}}$ from forming the non-switchable microcrystalline aggregates. Incorporating hydrating additives is usually effective in extending the lasting time of water prints. To improve the legible colourlasting time, various hygroscopic salts (that is, $\mathrm{NaCl}, \mathrm{CaCl}_{2}$, $\mathrm{NaNO}_{3}, \mathrm{CH}_{3} \mathrm{COONa}$ and $\mathrm{CH}_{3} \mathrm{COONH}_{4}$ ) have been investigated for the $\mathbf{R P}$, and $\mathrm{NaNO}_{3}$ was the one selected in our system. The four layers of the RP structure (Fig. 4a) include: (i) the bottom layer consisting of the paper substrate, (ii) a passivation layer consisting of PEG directly in contact with the paper substrate as a means to prevent hydrogen bonding between $\mathbf{1}_{\mathrm{C}}$ and the exposed donors on the paper surface, (iii) a thin film of PEG in which $\mathbf{1}_{\mathrm{C}}$ is 'dissolved' serving as the imaging layer along with $\mathrm{NaNO}_{3}$ as the hydrating additive to extend the image lifetime and (iv) a final layer of PEG coated on the surface acting as an overcoat to prevent not only the undesired colouration caused by a damp atmosphere but also problems of any possible dye leakage and potential dye exposure upon human contact. We have successfully printed on the paper by water-jet printing using a commercially available ink-jet printer with a cartridge filled purely with water (Fig. 4b). At present, the minimum amount of water for the colour appearance of the prints in our water-jet printing system is found to be $12 \mathrm{pl}$ per colour dot based on HP deskjet 2010 printer (see Supplementary Fig. 9). Good resolution of the blue prints in size 10.5 has been achieved so far with current water-jet printing, and the legibility of the prints can last $22 \mathrm{~h}$ under ambient condition $\left(\mathrm{T}<35^{\circ} \mathrm{C}\right.$, humidity $\left.20-80 \%\right)$, which is long enough for the purposes of temporary reading. The
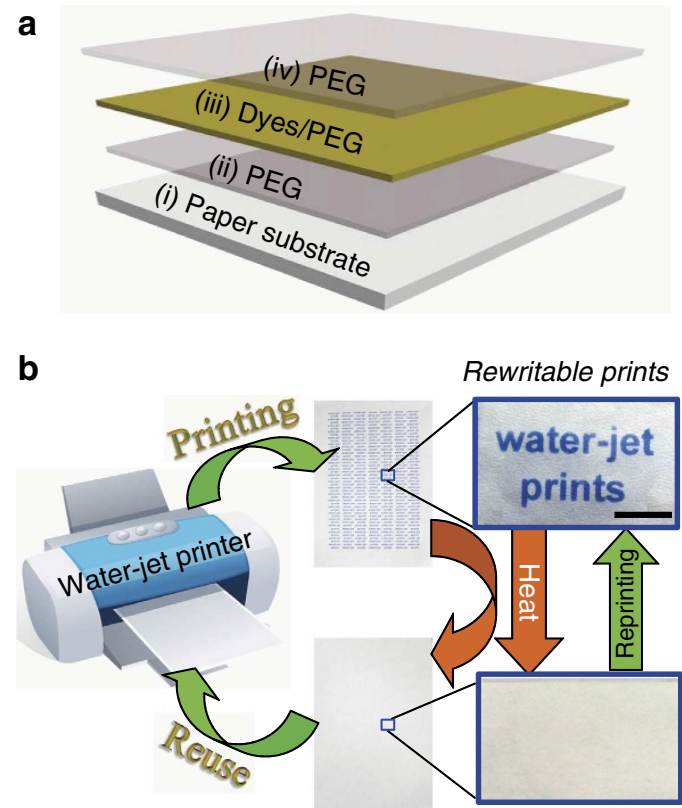

Figure 4 | Structural design and water-jet printing of RP. (a) Schematic illustrations of the four-layer structure used to create the RP materials based on 1. (i) Filter paper is used as the substrate layer; (ii) PEG is used as the passivation layer; (iii) a thin film of PEG in which $\mathbf{1}_{\mathbf{c}}$ is dissolved serves as the imaging layer; (iv) a film of PEG coated on top acts as a protection layer. (b) Schematic illustrations of a printing-reuse cycle of paper based on water-jet RP. Photographs of the letters produced by water-jet printing on this RP (above) followed erasing of those images by mild heating (below). Scale bar $=5 \mathrm{~mm}$. rate of the colour developing with water is found to be in less than $1 \mathrm{~s}$, and this means that the colour appears instantly during the water-jet printing from a practical perspective. The prints can also be erased quickly by mild heating. The rates of colour disappearance varied with the heating temperature, and it takes about $30 \mathrm{~s}$ to vanish the colour prints at $70^{\circ} \mathrm{C}$. Dedicated efforts are being given to further improve both resolution and imagelasting time.

Reversibility and colour variety of the rewritable prints. Reflective UV-vis spectroscopy was used to test the reversibility and repeatability. In the absence of water, the RP material exhibits a hump absorption band centred around $301 \mathrm{~nm}$, which is the characteristic feature of $\mathbf{1}_{\mathrm{C}}$. Upon introduction of water, a new absorption band centred at $592 \mathrm{~nm}$ associated with $\mathbf{1}_{\mathbf{O}}$ is observed, accompanied by the diminishment of the one centred at $301 \mathrm{~nm}$ (Fig. 5a). The reflectivity at $592 \mathrm{~nm}$ was recorded after repetitive writing with water spraying and erasing by heating at $70^{\circ} \mathrm{C}$. It is found that only a slight decrease in colour intensity is observed after more than 10 consecutive write-erase cycles

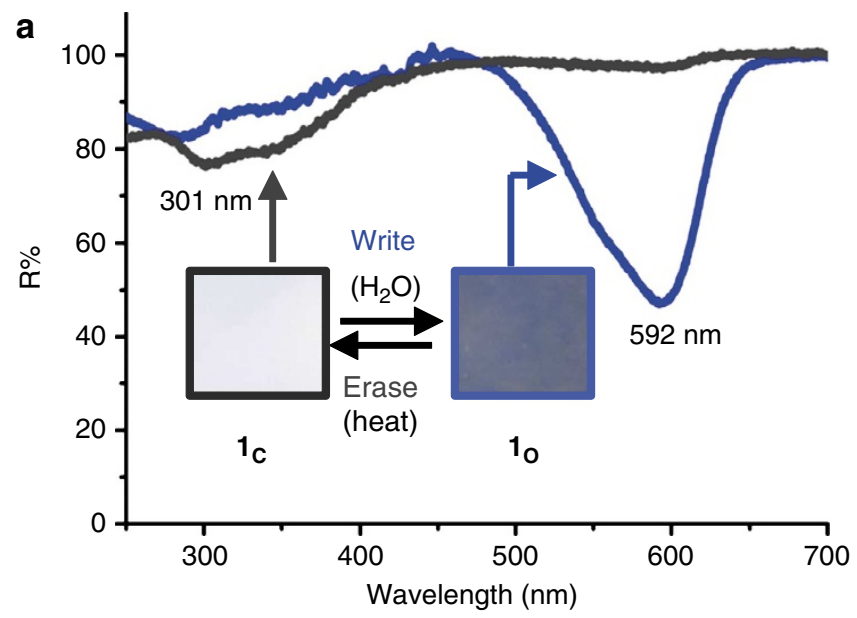

b

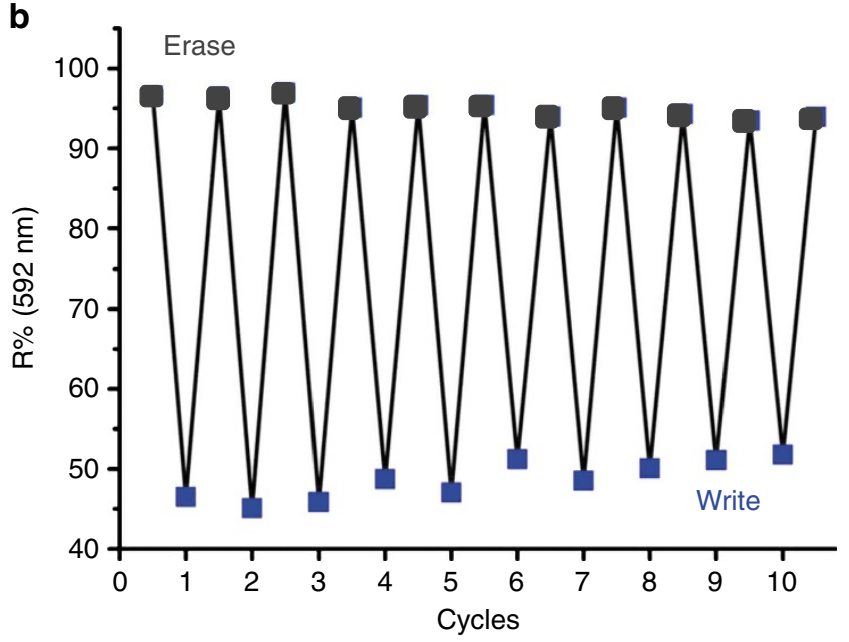

Figure 5 | Reversibility and repeatability of hydrochromic RP. (a) The reflective UV-vis spectra of the RP integrated with $\mathbf{1}$ before (black trace) and after (blue trace) addition of water, at which points the material turns from a white to a dark blue, respectively. The size of the photographs for rewritable papers is $40 \mathrm{~mm}$ by $40 \mathrm{~mm}$. (b) A plot of the reflectivity at $592 \mathrm{~nm}$ versus the number of cycles as the $\mathbf{R P}$ material is cycled through water spraying (write) and water removal (erase) by means of heating at $70^{\circ} \mathrm{C}$. 
(Fig. 5b), and no colour can be detected from the back side of the paper after dozens of rewritings. This indicates that the reversible hydrochromic switching behaviour of $\mathbf{1}$ can be retained in the RP material, and $\mathbf{1}$ can be immobilized in the imaging layer without leaking.

Different colours of RP can also be achieved using suitable hydrochromic dyes. For example, the oxazolidines 2, 3 and 4 can also be reversibly switched to their CFs by water, which exhibit magenta, gold and purple colours when stimulated with water, respectively (see Supplementary Fig. 10). After integration of these dyes using similar procedures to $\mathbf{1}$ on the paper substrate, RPs showing magenta, gold and purple characters can be obtained by water-jet printing (Fig. 6). At present, our

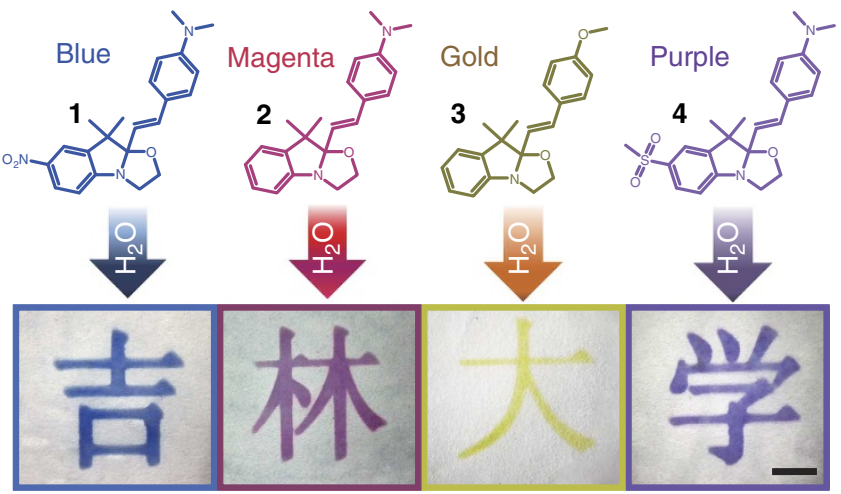

Figure 6 | Water-jet prints with different colours. Different colours of RPs can be achieved by integration of $\mathbf{1 - 4}$, respectively. The four Chinese characters shown, which mean Jilin University, were printed by waterjetting. Scale bar $=1 \mathrm{~cm}$. rewritable papers are in the monochromic stage of development. Multicolour rewritable paper with longer image lifetime is currently under investigation.

\section{Discussion}

On the basis of the knowledge on relationship of the molecular structures to their hydrorchromic properties obtained from this study, we can anticipate that halochromic oxazines with appropriate functional groups pioneered by Professor F.M. Raymo's group ${ }^{37-39}$ may also be used as hydrochromic materials.

As regards to toxicity and safety issues of the rewritable materials in specific and extreme exposure situations, the cytotoxicity tests of 1-4 have been conducted in HeLa cells using MTT assays. Results from $24 \mathrm{~h}$ incubation tests indicate that the hydrochromic dyes we used here are within low toxicity range (see Supplementary Fig. 11). Toxicological tests over extended periods of exposure to the dyes with mice are in progress, and these results will be reported later as a continuation of this work. We need to point out that, as the dyes we used here are insoluble in water, the potential problem of dye exposure upon human contact is eliminated after a non-toxic PEG-capping layer is used for the RP material.

The current rewritable prototype is easily compatible with commercially available ink-jet printers by simply replacing ink with water in the cartridges. This simple water-refilling solves the primary problem of frequent replacement of ink cartridges associated with ink-jet users. To demonstrate the feasibility of our developed RP for the practical applications, we printed an A4 size water-jet prints in comparison with HP ink-jet prints (Fig. 7). The legibility and resolution of our current water-jet prints seem good enough for general reading purpose, and we are confident

b

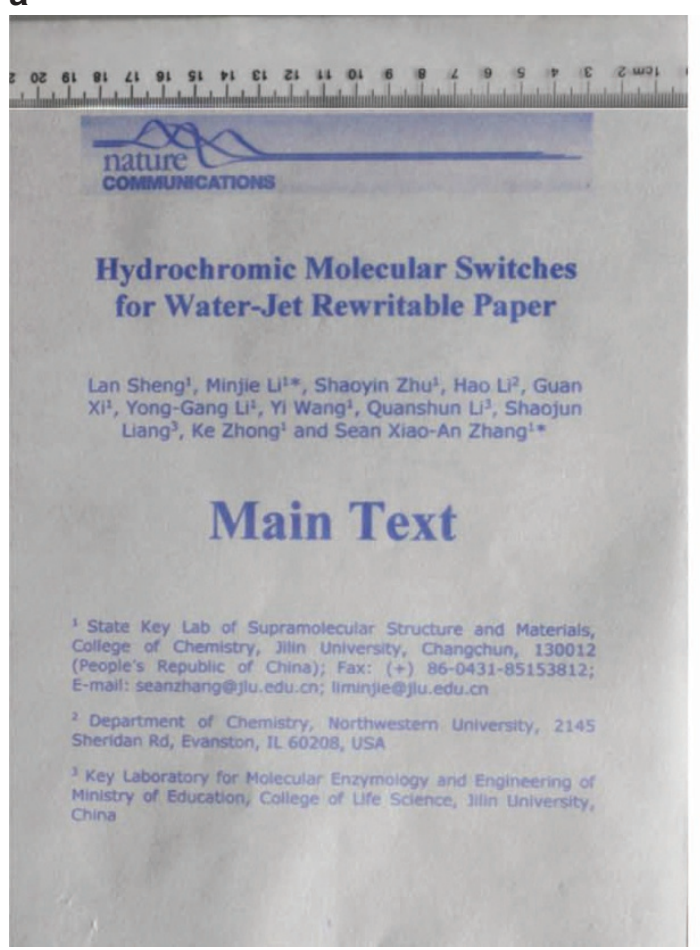

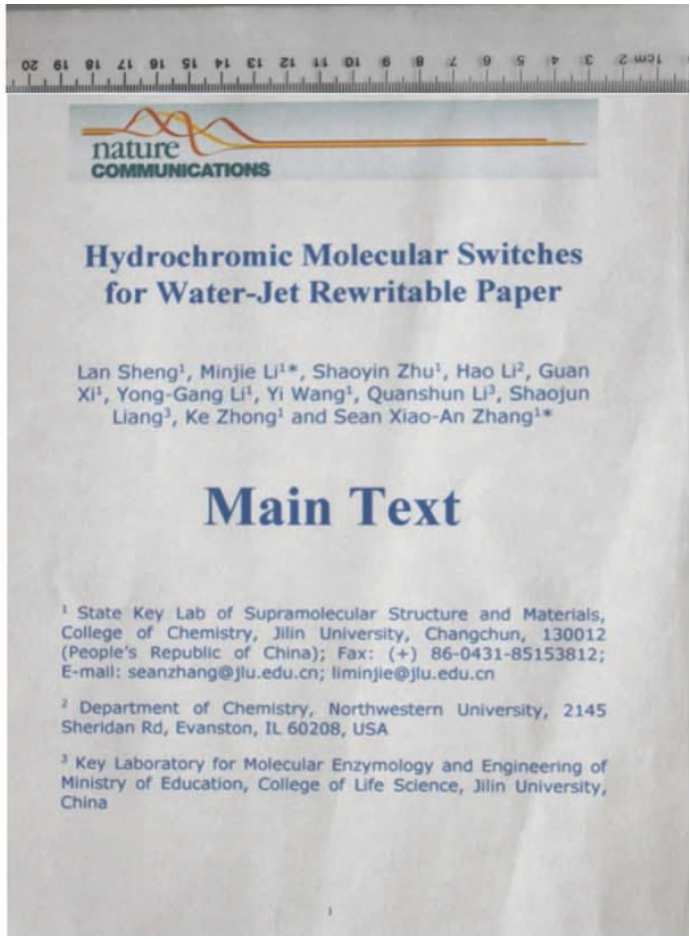

Figure 7 | Side-by-side comparison of water-jet prints with ink-jet prints. (a) Water-jet prints $(210 \times 297 \mathrm{~mm})$ printed by HP DeskJet 2010 printer with cartridges (HP 704) refilled with water $(4 \mathrm{ml})$ on our RP based on dye 1. (b) Ink-jet prints $(210 \times 297 \mathrm{~mm})$ printed by the same printer with commercial HP 704 black and tricolour cartridges. 
to improve further the colour intensity and evenness of our rewritable media.

According to a rough estimation, the cost per print of the RP (based on a conservative 10 times reusage/sheet) would be approximately one-seventeenth of the normal ink-jet print (see Supplementary Table 2). Even though the $22 \mathrm{~h}$ legible imagelasting time accomplished so far is not long enough for keeping records, this still provides a good alternative for reducing paper waste from temporary reading. Furthermore, our rewritable paper overcomes some disadvantages of the current version of rewritable media, including higher cost and more energy consumption.

In summary, we present a green and economic strategy to develop ink-free rewritable paper by introducing hydrochromic dyes in paper and using water as the sole trigger. Important insights on the relationship of the molecular structures with their hydrorchromic properties have been obtained, which allow us to screen and identify quickly the ideal candidates to be used for the imaging materials. Negative impacts on the practical usage of the rewritable dyes from the fixed spatial polyhydroxyl environment of the paper, microcrystalline aggregation and excessive ambient moisture have been successfully overcome. A prototype of inkfree RP with legibility lasting nearly one full day per print under normal ambient conditions has been developed, and dozens of write-erase cycles without obvious colour fading have been realized. This water-jet rewritable printing is pointing a very good direction on green life, and will inspire further development of better rewritable media for global sustainable growth.

\section{Methods}

Synthesis of compounds 1-10. General procedure I for synthesis of A1-10: The phenylhydrazines (1 equivalent (eq.), 3-methyl-2-butanones (1.3 eq.) and $\mathrm{H}_{2} \mathrm{SO}_{4}$ (0.1 eq.) were dissolved in glacial acetic acid and then heated under reflux for $7.5 \mathrm{~h}$ under nitrogen. After the reaction was cooled to room temperature, the solvent was evaporated in vacuo. The residue was dissolved in $\mathrm{CH}_{2} \mathrm{Cl}_{2}$, washed with $10 \%$ aqueous $\mathrm{Na}_{2} \mathrm{CO}_{3}$ and dried with anhydrous $\mathrm{Na}_{2} \mathrm{SO}_{4}$. Then $\mathrm{CH}_{2} \mathrm{Cl}_{2}$ was removed in vacuo. The products were obtained using vacuum distillation or purified using column chromatography to afford A1-10.

General procedure II for synthesis of B1-10: A1-10 (1 eq.) and 2-bromoethanol (1.2 eq.) were dissolved in $\mathrm{MeCN}$ and heated under reflux for $25 \mathrm{~h}$ under nitrogen, and then the reaction was allowed to cool to room temperature. The precipitate was filtered and washed with $\mathrm{Et}_{2} \mathrm{O}$. The resulting solids were collected and recrystallized from EtOH/EtOAc to afford B1-10.

General procedure III for synthesis of C1-10: B1-10 (1 eq.) and aromatic aldehydes (D1-10) (1.2 eq.) were dissolved in $\mathrm{EtOH}$ under nitrogen with the addition of three drops of $\mathrm{CH}_{3} \mathrm{SO}_{3} \mathrm{H}$ as a catalyst. The solution was maintained at reflux for $6 \mathrm{~h}$ before stopping the heat. The reaction was then allowed to cool to room temperature. The resulting precipitate was washed by EtOAc to afford crude products. The crude products were recrystallized from EtOAc/hexane to afford C1-10 as solids.

General procedure IV for synthesis of 1-10: C1-10 were basified by adding 5\% $\mathrm{NaHCO}_{3}$ aqueous solution until the solution turned brown, at which point the solutions were extracted with EtOAc. The organic layers were collected, dried over anhydrous $\mathrm{Na}_{2} \mathrm{SO}_{4}$ and concentrated to afford 1-10 as solids.

Preparation of rewritable papers. The rewritable paper integrated with $\mathbf{1}$ was prepared in a layer-by-layer manner. The filter paper substrate was coated with a layer of $10 \mathrm{wt} \% \mathrm{PEG}$ aqueous solution and dried at $70^{\circ} \mathrm{C}$. Then $\mathrm{EtOH} / \mathrm{H}_{2} \mathrm{O}(2 / 3$ by volume) solution of $1\left(0.08 \mathrm{mmoll}^{-1}\right)$ containing $6 \mathrm{wt} \% \mathrm{PEG}$ and $0.01 \mathrm{wt} \%$ of $\mathrm{NaNO}_{3}$ as the hydrating agent is coated over the initial PEG layer. Lastly, another protective layer of PEG was coated on the top using 10\% PEG aqueous solution after the first two layers were dried completely. Layer-by-layer coating methods and procedures as these used for $\mathbf{1}$ were used for the fabrication of rewritable paper based on 2, 3 and 4. Owing to the different sensitivities of these molecules to the hydroxyl groups on the surface of the filter paper, the contents of each passivation layer varied slightly. The passivation layer for 2 was prepared from a $10 \%$ PEG aqueous solution with $1 \mathrm{mmoll}^{-1} \mathrm{NaHCO}_{3}$. The passivation layer for 3 was prepared from a 10\% PEG aqueous solution. The passivation layer for 4 was prepared from a $20 \%$ PEG aqueous solution. PEG 20000 (molecular weight: 17,000-22,000) was purchased from GUANGFU fine chemical research institute (Tianjin, China). Cellulose filter paper (Whatman-Xinhua, grade 91, Hangzhou, China) is selected as the paper substrate.
Cell cytotoxicity test condition. HeLa cells (a human epithelial cervical cancer cell line) were cultivated at $37^{\circ} \mathrm{C}$ under humidified $5 \% \mathrm{CO}_{2}$ in Dulbecco's modified Eagle's medium, supplemented with $10 \%$ fetal bovine serum and $100 \mu \mathrm{g} \mathrm{ml}{ }^{-1}$ penicillin-streptomycin. HeLa cells were seeded in 96-well tissue culture plates at a density of 5,000 per well in Dulbecco's modified Eagle's medium containing 10\% fetal bovine serum. The cytotoxicity of the dyes was evaluated by determining cell viability after $48 \mathrm{~h}$ of incubation with various final concentrations of 1-4 $\left(0.3-30 \mu \mathrm{g}\right.$ of dyes $\left.\mathrm{ml}^{-1}\right)$. The number of viable cells was determined by estimating their mitochondrial reductase activity using the tetrazolium-based colorimetric method (MTT conversion test) with a Microplate Reader (GF-M3000, Caihong, China) at a wavelength of $495 \mathrm{~nm}$. The relative cell viability (\%) for the controls (containing cell culture medium) was calculated as: test/control $\times 100$.

\section{References}

1. Sarantis, H. Business Guide to Paper Reduction (ForestEthics, 2002)

2. Kishimura, A., Yamashita, T., Yamaguchi, K. \& Aida, T. Rewritable phosphorescent paper by the control of competing kinetic and thermodynamic self-assembling events. Nat. Mater. 4, 546-549 (2005).

3. Yoon, B. et al. Inkjet printing of conjugated polymer precursors on paper substrates for colorimetric sensing and flexible electrothermochromic display. Adv. Mater. 23, 5492-5497 (2011).

4. Mortimer, R. J., Dyer, A. L. \& Reynolds, J. R. Electrochromic organic and polymeric materials for display applications. Displays 27, 2-18 (2006).

5. Hays, D. A. Paper documents via the electrostatic control of particles. J. Electrostat. 51, 57-63 (2001).

6. Kobayashi, N., Miura, S., Nishimura, M. \& Urano, H. Organic electrochromism for a new color electronic paper. Sol. Energy Mater. Sol. Cells 92, 136-139 (2008).

7. Comiskey, B., Albert, J. D., Yoshizawa, H. \& Jacobson, J. An electrophoretic ink for all-printed reflective electronic displays. Nature 394, 253-255 (1998).

8. Hayes, R. A. \& Feenstra, B. J. Video-speed electronic paper based on electrowetting. Nature 425, 383-385 (2003).

9. Ge, J., Goebl, J., He, L., Lu, Z. \& Yin, Y. Rewritable photonic paper with hygroscopic salt solution as ink. Adv. Mater. 21, 4259-4264 (2009).

10. Fudouzi, H. \& Xia, Y. Photonic papers and inks: color writing with colorless materials. Adv. Mater. 15, 892-896 (2003).

11. Hermy, M., Honnay, O., Firbank, L., Bokdam, C. G. \& Lawesson, J. E. An ecological comparison between ancient and other forest plant species of Europe, and the implications for forest conservation. Biol. Conserv. 91, 9-22 (1999).

12. Horiguchi, T., Koshiba, Y., Ueda, Y., Origuchi, C. \& Tsutsui, K. Reversible coloring/decoloring reaction of leuco dye controlled by long-chain molecule. Thin Solid Films 516, 2591-2594 (2008).

13. Yamamoto, S., Furuya, H., Tsutsui, K., Ueno, S. \& Sato, K. In situ observation of thermochromic behavior of binary mixtures of phenolic long-chain molecules and fluoran dye for rewritable paper application. Cryst. Growth Des. 8, 2256-2263 (2008).

14. Kawashima, I., Takahashi, H., Hirano, S. \& Matsushima, R. A photon-mode full-color rewritable image using photochromic compounds. J. Soc. Inf. Display 12, 81-85 (2004).

15. Kohno, Y., Tamura, Y. \& Matsushima, R. Simple full-color rewritable film with photochromic fulgide derivatives. J. Photochem. Photobiol. A 201, 98-101 (2009).

16. Zhang, J. Z., Schwartz, B. J., King, J. C. \& Harris, C. B. Ultrafast studies of photochromic spiropyrans in solution. J. Am. Chem. Soc. 114, 10921-10927 (1992).

17. Khairutdinov, R. F. et al. Photochromism of spirooxazines in homogeneous solution and phospholipid liposomes. J. Am. Chem. Soc. 120, 12707-12713 (1998).

18. Tomasulo, M., Sortino, S. \& Raymo, F. M. A fast and stable photochromic switch based on the opening and closing of an oxazine ring. Org. Lett. 7, 1109-1112 (2005).

19. Mancois, F. et al. Two-way molecular switches with large nonlinear optical contrast. Chem. Eur. J. 15, 2560-2571 (2009).

20. Lee, J., Lee, C. W. \& Kim, J. M. Fabrication of patterned images in photochromic organic microfibers. Macromol. Rapid Commun. 31, 1010-1014 (2010).

21. Kinashi, K., Horiguchi, T., Tsutsui, K., Ishida, K. \& Ueda, Y. Reversible multi-coloring reaction of spironaphtooxazine controlled by long-chain molecule. J. Photochem. Photobiol. A 213, 189-193 (2010).

22. Hirshberg, Y. \& Fischer, E. Photochromism and reversible multiple interna transitions in some spiropyrans at low temperatures. Part II. J. Chem. Soc. 3129-3137 (1954).

23. Minkin, V. I. Photo-, thermo-, solvato-, and electrochormic spiroheterocyclic compounds. Chem. Rev. 104, 2751-2776 (2004).

24. Sun, X. D., Fan, M. G., Meng, X. J. \& Knobbe, E. T. Acidichromic effects in spiro (1,3,3-trimethylindolo-2,3'-naphth[1,2-b]-1,4-oxazine), a photochromic 
compound I. Absorption characteristics. J. Photochem. Photobiol. A 102, 213-216 (1997).

25. Raymo, F. M. \& Giordani, S. Signal processing at the molecular level. J. Am. Chem. Soc. 123, 4651-4652 (2001).

26. Woodruff, A., Hasbrouck, J. \& Augustin, S. Proceedings, Green Day CHI (A bright green perspective on sustainable choices) 5-10 April, 313 (Florence, Italy, 2008).

27. Shiraishi, Y., Itoh, M. \& Hirai, T. Thermal isomerization of spiropyran to merocyanine in aqueous media and its application to colorimetric temperature indication. Phys. Chem. Chem. Phys. 12, 13737-13745 (2010).

28. Gaeva, E. B. et al. Solvation effects on spirooxazine to merocyanine thermal reversion kinetics in acetonitrile-water binary mixtures. J. Phys. Org. Chem. 18, 315-320 (2005).

29. Burke, K., Riccardi, C. \& Buthelezi, T. Thermosolvatochromism of nitrospiropyran and merocyanine free and bound to cyclodextrin. J. Phys. Chem. B 116, 2483-2491 (2012).

30. Keum, S.-R. et al. Unusual reverse photochromic behavior of indolinobenzospiropyran 6-carboxylates in aqueous binary solvents. Bull. Korean Chem. Soc. 27, 187-188 (2006).

31. Song, X., Zhou, J., Li, Y. \& Tang, Y. Correlations between solvatochromism, Lewis acid-base equilibrium and photochromism of an indoline spiropyran. J. Photochem. Photobiol. A 92, 99-103 (1995).

32. Sanguinet, L. et al. Champagne, B. Acido- and phototriggered NLO properties enhancement. J. Phys. Chem. B 109, 11139-11150 (2005).

33. Zhu, S. et al. A spirooxazine derivative as a highly sensitive cyanide sensor by means of UV-visible difference spectroscopy. Analyst 137, 5581-5585 (2012).

34. Tomasulo, M., Sortino, S., White, A. J. P. \& Raymo, F. M. Fast and stable photochromic oxazines. J. Org. Chem. 70, 8180-8189 (2005).

35. Berman, E., Fox, R. E. \& Thomson, F. D. Photochromic spiropyrans. I. The effect of substituents on the rate of ring closure. J. Am. Chem. Soc. 81, 5605-5608 (1959)

36. Liu, R., Zhao, F. \& Deng, F. Synthesis of nitro derivatives of indolinospirobenzopyrans. Youji Ниахие 6, 440-443 (1987).
37. Deniz, E., Tomasulo, M., Sortino, S. \& Raymo, F. M. Substituent effects on the photochromism of bichromophoric oxazines. J. Phys. Chem. C 113, 8491-8497 (2009).

38. Tomasulo, M. \& Raymo, F. M. Use of oxazine compounds for making chromogenic materials. US 8,304,537 (2012).

39. Deniz, E. et al. Photoactivatable fluorophores for super-resolution imaging based on oxazine auxochromes. J. Phys. Chem. C 116, 6058-6068 (2012).

\section{Acknowledgements}

S.X.-A.Z. and M.L. thanks the Jilin University, State Key Lab of Supramolecular Structure and Materials for startup support. Acknowledgment is also made to the National Science Foundation of China (Grant No. 21072025 and 51001020) for support of this research.The authors also thank Albert C Fahrenbach from Northwestern University (USA) for helpful scientific discussions and for his aid in preparation of the manuscript.

\section{Author contributions}

S.X.-A.Z, L.S. and M.L. conceived and designed the experiments. L.S., S.Z. and Y.W. synthesized the compounds. L.S. performed the experiments. G.X., Y.-G.L. and K.Z. helped to prepare the RP materials. H.L. contributed to NMR experimental data and analysis. Q.L. and S.L. did and analysed the cell cytotoxicity experiments. L.S., H.L., M.L. and S.X.-A.Z. analysed the results and wrote the manuscript.

\section{Additional information}

Supplementary Information accompanies this paper at http://www.nature.com/ naturecommunications

Competing financial interests. The authors declare no competing financial interests.

Reprints and permissions information is available online at http://npg.nature.com/ reprintsandpermissions/

How to cite this article: Sheng, L. et al. Hydrochromic molecular switches for water-jet rewritable paper. Nat. Commun. 5:3044 doi: 10.1038/ncomms4044 (2014). 